\title{
Transcriptomic characterization of the molecular mechanisms induced by RGMa during skeletal muscle nuclei accretion and hypertrophy
}

\author{
Aline Gonçalves Lio Copola', Íria Gabriela Dias dos Santos' ${ }^{1}$, Luiz Lehmann Coutinho², \\ Luiz Eduardo Vieira Del-Bem³ ${ }^{3}$ Paulo Henrique de Almeida Campos-Junior ${ }^{4}$, \\ Izabela Mamede Costa Andrade da Conceição ${ }^{5}$, Júlia Meireles Nogueira ${ }^{1}$, Alinne do Carmo Costa ${ }^{1}$, \\ Gerluza Aparecida Borges Silva ${ }^{1}$ and Erika Cristina Jorge ${ }^{1^{*}}$
}

\begin{abstract}
Background: The repulsive guidance molecule a (RGMa) is a GPI-anchor axon guidance molecule first found to play important roles during neuronal development. RGMa expression patterns and signaling pathways via Neogenin and/ or as BMP coreceptors indicated that this axon guidance molecule could also be working in other processes and diseases, including during myogenesis. Previous works from our research group have consistently shown that RGMa is expressed in skeletal muscle cells and that its overexpression induces both nuclei accretion and hypertrophy in muscle cell lineages. However, the cellular components and molecular mechanisms induced by RGMa during the differentiation of skeletal muscle cells are poorly understood. In this work, the global transcription expression profile of RGMa-treated C2C12 myoblasts during the differentiation stage, obtained by RNA-seq, were reported.
\end{abstract}

Results: RGMa treatment could modulate the expression pattern of 2,195 transcripts in C2C12 skeletal muscle, with 943 upregulated and 1,252 downregulated. Among them, RGMa interfered with the expression of several RNA types, including categories related to the regulation of RNA splicing and degradation. The data also suggested that nuclei accretion induced by RGMa could be due to their capacity to induce the expression of transcripts related to 'adherens junsctions' and 'extracellular-cell adhesion', while RGMa effects on muscle hypertrophy might be due to (i) the activation of the mTOR-Akt independent axis and (ii) the regulation of the expression of transcripts related to atrophy. Finally, RGMa induced the expression of transcripts that encode skeletal muscle structural proteins, especially from sarcolemma and also those associated with striated muscle cell differentiation.

Conclusions: These results provide comprehensive knowledge of skeletal muscle transcript changes and pathways in response to RGMa.

Keywords: Axon Guidance, Myogenesis, Hypertrophy, Hyperplasia, Skeletal muscle differentiation, Transcriptomic analysis

\footnotetext{
*Correspondence: ecjorge@ufmg.br; erika.cris.jorge@gmail.com ${ }^{1}$ Departamento de Morfologia, Instituto de Ciências Biológicas, Universidade Federal de Minas Gerais, Av Antonio Carlos, 6627, Pampulha, Belo Horizonte, Minas Gerais 31.270-901, Brasil

Full list of author information is available at the end of the article
}

\section{Background}

Repulsive guidance molecule a (RGMa) comprises the first repulsive glycoprotein member identified in the family of repulsive guidance molecules [1]. It was originally 
identified as a repulsive clue in the orientation of axonal growth in the central and peripheral nervous system and as an important target for neuronal survival [1-4] However, RGMa action domains were found to go beyond the processes related to neurogenesis and could be extended to different processes, including the induction of endochondral ossification during skeletal development [5], the suppression of endothelial tube formation [6], and inflammatory responses $[7,8]$.

These diverse functions can be performed by RGMa because it can signal through different receptors and work as a modular protein. The RGMa C-terminal domain (C-RGMa) harbours a GPI-anchor and presents affinity to the type I transmembrane neogenin receptor $[9,10]$, which is known as a guidance receptor for migrating neuronal and mesodermal cells [11-13]. This domain also harbours a von Willibrand type D structural domain, containing a GDPH autocatalytic site [14]. The RGMa N-terminal domain (N-RGMa) harbours a signal peptide, an additional neogenin-binding site, and an RGD motif, that is known to be important in cell-cell adhesion processes mediated by integrins [15]. However, RGMa signaling through integrins has not been reported thus far. Notably, N-RGMa presents high affinity to bone morphogenetic proteins (BMP) ligands, making RGMa (and all the members of this family) a modulator of this important signaling pathway [16-19]. N-RGMa shares the same binding site on the BMP ligand with the ectodomain of the BMP type I receptor A (BMP-R1A), meaning that RGM can induce the BMP canonical signaling pathway via activation of Smad 1/5/8. RGMa could also integrate neogenin and BMP signaling cascades [5, 20-22]. Finally, RGMa was recently found to promote astrogliosis and glial scar formation in a rat model of middle cerebral artery occlusion/reperfusion by forming a complex with ALK5 and Smad2/3, which are the main members of the transforming growth factor $\beta 1$ (TGF $\beta 1$ ) signaling pathway [23].

In previous works, we found $R G M a$ transcripts in the myogenic and satellite cell precursors in the somites during chicken embryonic development [24] and at the sarcolemma and in the sarcoplasm of adult mice muscle cells [25]. RGMa overexpression in C2C12 cells induced the formation of larger myotubes (hypertrophy) with an increased number of myonuclei (nuclei accretion), while its knockdown resulted in the appearance of smaller cells, with a deficient ability to form multinucleated myotubes [25].

Skeletal muscle cell size is known to be determined by the balance between protein and cellular turnover [26-28]. Because of cellular turnover, the skeletal muscle cell grows by myonuclei accretion, in a process mediated by cell fusion. The increase of myonuclei into myofibers leads to muscle mass expansion due to the higher rate of transcription given the nuclear turnover [29]. Muscle nuclei accretion is important not only during embryonic development but also during muscle regeneration [29-36]. In contrast, because of protein turnover, the skeletal muscle cell grows by upregulating protein synthesis pathways, consequently increasing the level of protein within the muscle tissue [27, 29]. Although hypertrophy and nuclei accretion are two distinct processes, they frequently occur together [36], and not all signals involved during the proliferation and differentiation of skeletal musculature are known.

Despite having found that RGMa can induce hypertrophy and nuclear accretion in skeletal muscle cells cultivated in vitro, the molecular mechanisms that are induced by this axon guidance molecule in these particular cells have not been investigated thus far. Our hypothesis is that RGMa can modulate the expression of a number of transcripts in skeletal muscle cells, especially those involved with nuclei accretion and striated muscle cell differentiation. In this work, $\mathrm{C} 2 \mathrm{C} 12$ cells were treated with RGMa recombinant protein to investigate the molecular mechanisms that are modulated by this axon guidance molecule during myogenic differentiation. This was the first work to show, through RNA-seq analysis, the transcript targets and molecular profile triggered by RGMa during skeletal muscle differentiation and its possible involvement in multiple functions, including cell fusion and hypertrophy.

\section{Results}

Overview of the RNA-seq data and differentially expressed transcripts (DETs)

The quality of the generated sequence database was first evaluated to verify the internal consistency and reproducibility of the replicate samples, as well as the disparity among them. The Pearson correlation coefficient (PCC) of the normalized read-counts revealed a perfect positive linear correlation between all RGMa-treated samples and an extremely strong correlation among the control ones (Fig. 1A). The analysis also revealed a subtle difference between treated and control samples, as there was a positive linear correlation showing Pearson $r$ coefficients above 0.97 among all correlated samples (Fig. 1B). MA-plot analysis revealed that RGMa treatment modulated gene expression in skeletal muscle cells, with very few of them presenting a drastic effect (Fig. 1C).

The expression of 23,855 transcripts could be detected after normalization, and 2,195 were found as differentially expressed transcripts (DETs, $p<0.05$, Fig. 1B, grey dots), with 943 upregulated and 1,252 downregulated by RGMa treatment compared to the control (Fig. 1C, blue dots with $\log 2(\mathrm{FC})>0$ and $\log 2(\mathrm{FC})<0$, respectively). 
Twenty-six DETs were exclusively expressed in RGMatreated myoblasts, and 79 DETs had their expression drastically altered by the treatment (Supplementary Table 1). Differential expression analysis was also performed in gene level. We found that RGMa could modulate the expression of 1,788 genes (DEGs, $p<0.05$, Supplementary Table 2). From the 2,195 DETs, 1,091 were also found as DEGs, meaning that $1,104(\sim 50 \%)$ were found as differentially expressed only at the transcription level.

The most drastic effects among the DETs were also observed as a heatmap of transcripts with enriched muscle-associated terms (Fig. 1D). The heatmap also allowed the observation that the expression of the majority of the transcripts did not change considerably between the control and treated samples. The 20 most upregulated and 20 most downregulated transcripts by RGMa treatment in C2C12 cells are shown in Table 1.

The most highly upregulated DET induced by RGMa treatment was the Pou $2 F 1$ transcription factor (isoform Pou2F1-208, ENSMUST00000160260.9), also known as Oct-1 (Table 1). Among the other highly expressed genes, RGMa was able to induce the expression of genes related to skeletal muscle structure, including sarcomere and costamere organization (e.g., Ank3, Nrap and Parva), vesicle formation and trafficking (e.g., Myo5a, Iqsec1, Tbc1d25, Acap and Rab1a), and control of the cell cycle (Mau 2 and Scaper).

Notably, another isoform of the Pou $2 F 1$ transcription factor (Pou2F1-205, ENSMUST00000111427.9) was found to be one of the most downregulated genes by RGMa treatment (Table 1).

Among the others, the downregulation of genes from the same categories included those associated with regulators of muscle mass and structure, such as Tsc1 and Tnpo3, with the formation of clathrin-coated vesicles (Ap2a1, and Picalm), and with cell cycle progression and apoptosis (Pcgf1, Kifc3 and Cep164) (Table 1).

\section{RNA categories among DET}

Given the reliability of the transcriptome data, we next classified all 2,195 DETs by RNA biotypes to determine which were the main RNA categories influenced by RGMa treatment. Among the 1,252 DETs that were found to be downregulated, 917 (73.2\%) were protein coding, $246(19.6 \%)$ were processed transcripts, 67 (5.35\%) were NMDs, and $22(1.75 \%)$ were pseudogenes (Fig. 2). Among the 943 upregulated DETs, 786 (83.3\%) were protein coding, $115(12.2 \%)$ were processed transcripts, $36(3.8 \%)$ were NMDs, $5(0.5 \%)$ were pseudogenes, and $1(0.1 \%)$ was a TEC (Fig. 2). Overall, this data revealed that most of the RNA biotypes that were modulated by RGMa treatment were ORF-containing RNAs, while the remaining were composed of RNAs mainly associated with the regulation of gene expression, including the NMD category, which was composed of transcripts containing a premature stop codon, and processed transcripts, a category composed of lncRNA, ncRNA, antisense, and intron-retained RNAs.

\section{GO pathway enrichment analysis of the non-coding RNA found as DETs}

The non-coding RNA found as DETs that were upregulated by RGMa treatment were mostly involved with the 'regulation of RNA splicing,' 'stress fibre,' 'myoblast fusion,' and 'integrin binding' (Fig. 3A), while 'regulation of RNA transport' and 'peptide biosynthetic process' were enriched among the downregulated non-coding DETs (Fig. 3B).

\section{GO pathway enrichment analysis of the protein coding RNA found as DETs}

DETs were characterized based on the Gene Ontology (GO) terms to identify the pathways that were enriched among the up- and downregulated transcripts. The enriched GO terms for the protein coding upregulated DETs were mostly related to the following biological processes: 'morphogenesis', 'metabolism,' and 'developmental regulation of muscle cell' (Fig. 4A). Related to the cellular components, RGMa treatment could induce the upregulation of transcripts associated with 'cytoskeleton,' 'cell projection,' 'endomembrane system,' 'adherens junction,' 'nucleus', and 'nucleoplasm' (Fig. 4B); and related to molecular function, transcripts were grouped as 'nucleic acid binding,' 'transcription factor binding, and 'regulation of GTPase' and 'Ras GTPase activity' (Fig. 4C).

A different pattern was found with the classification of the protein coding downregulated DETs; terms related

(See figure on next page.)

Fig. 1 Quality and transcriptomic profile of RGMa-treated myoblasts during myogenic differentiation. A Experimental design. B Pearson Correlation Coefficient (PCC) analysis of normalized read-counts denoted a high internal consistency and reproducibility of treated and control replicates. $\mathbf{C}$ MA plot analysis showing the RNA-seq profile of the $\log _{2}$ (fold change) distributions of all DETs in the average of normalised counts. Each point represents one transcript. Those dots marked in blue were detected as differentially expressed at a $5 \%$ FDR with $\log _{2}(\mathrm{FC})>0$ (upregulated) and $\log _{2}(F C)<0$ (downregulated) after RGMa treatment. Transcripts with similar expression levels are represented around the horizontal line $(y=0)$. Dots that are outside the window are plotted as triangles. D Heatmap analysis of DET with muscle-associated terms ('cellular component,',biological process,' and 'molecular function') of Gene Ontology (GO). Transcripts with the lowest expression values are marked in red, median expression values in black, and the highest expression values in green 


\section{A}
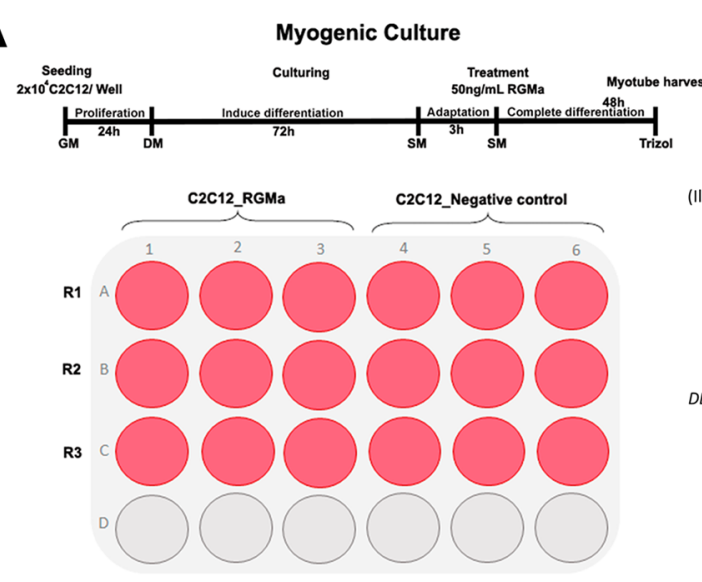

RNA sequencing

(IIlumina HiSeq2500 Paired end reads protocol)

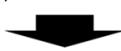

Trasnscriptomic Quantification (Salmon)

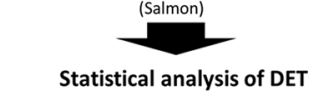

DEseq2 (Bioconductor) padj<0,05 Log2(FC) < $0 r>0$ Functional Analysis Cluego (Cytoscape) $\underbrace{}_{\substack{\text { RT_qPCR } \\ \text { 2-DDCt }}}$

B

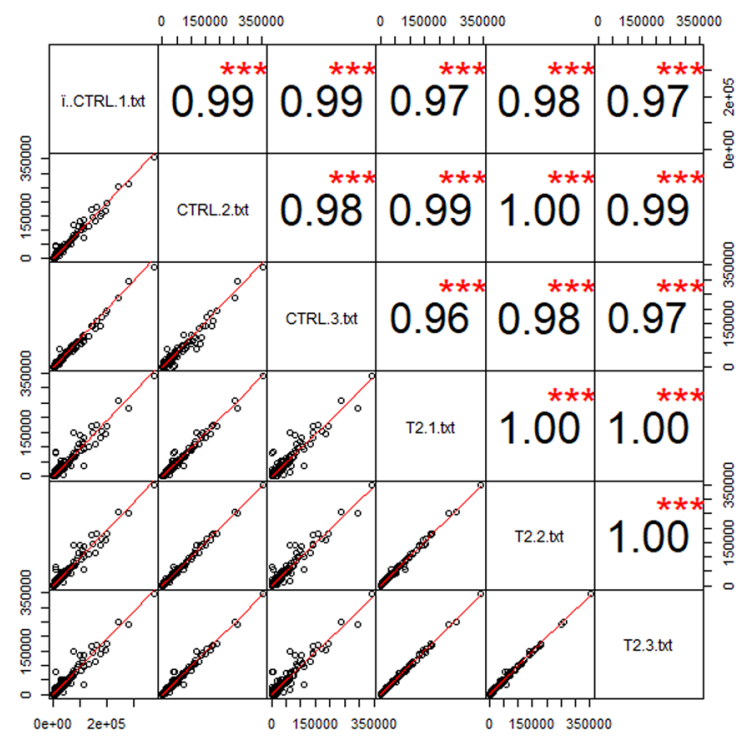

C
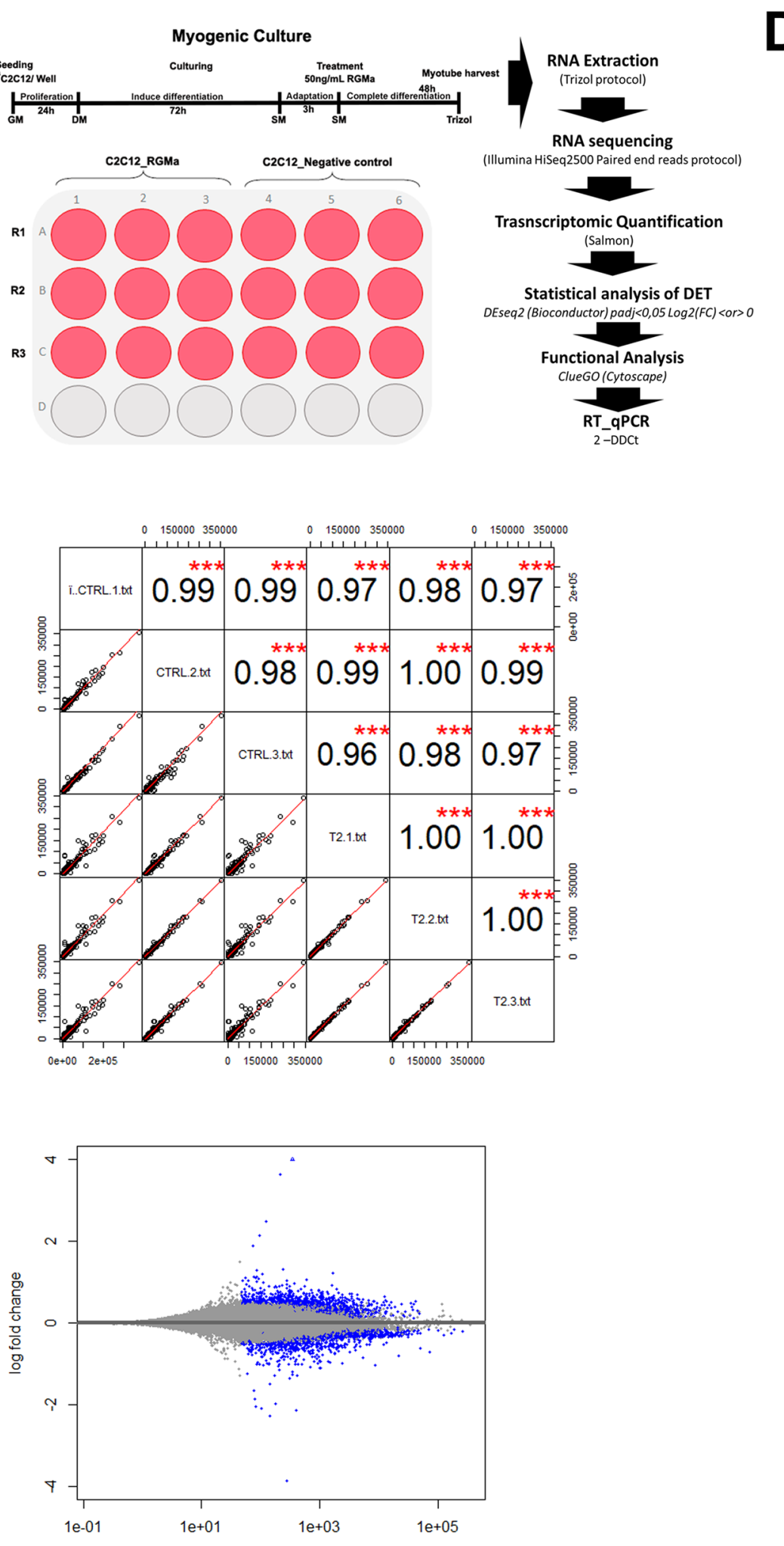

D

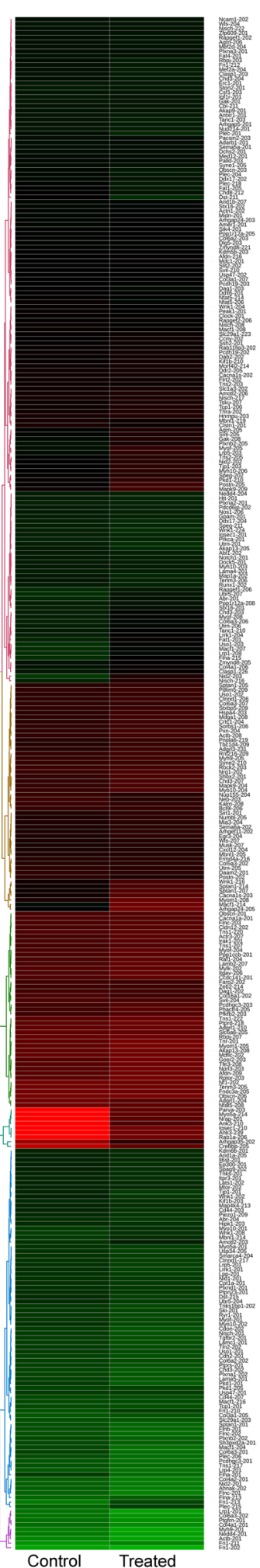

mean of normalized counts

Fig. 1 (See legend on previous page.) 
Table 1 The most drastically altered transcripts in RGMa-treated C2C12 myoblast, during myogenic differentiation

\begin{tabular}{|c|c|c|c|c|c|}
\hline \multicolumn{3}{|c|}{ Transcripts most downregulated by RGMa } & \multicolumn{3}{|c|}{ Transcripts most upregulated by RGMa } \\
\hline Ensembl Transcript Access & Trancript name & $\log 2(F C)<0$ & Ensembl Transcript Access & Trancript name & $\log 2(\mathrm{FC})>0$ \\
\hline ENSMUST00000113926.7 & $Z f x-203$ & $-12,09,700,663$ & ENSMUST00000160260.8 & Pou2f1-208 & $11,86,541,962$ \\
\hline ENSMUST00000187142.1 & Zfp469-202 & $-11,6,517,089$ & ENSMUST00000075836.11 & Dock7-202 & $11,30,650,103$ \\
\hline ENSMUST00000111427.8 & Pou2f1-205 & $-11,58,532,487$ & ENSMUST00000182593.7 & Prrc2c-209 & $11,18,773,896$ \\
\hline ENSMUST00000113870.2 & Tsc1-204 & $-11,08,073,619$ & ENSMUST00000182155.7 & Ank3-210 & $10,39,693,989$ \\
\hline ENSMUST00000169353.2 & Kifc3-202 & $-10,96,464,702$ & ENSMUST00000040711.14 & Nrap-201 & $10,01,424,135$ \\
\hline ENSMUST00000177916.7 & Zfp131-201 & $-10,66,135,536$ & ENSMUST00000106643.7 & Parva-203 & $9,856,496,026$ \\
\hline ENSMUST00000134230.7 & Hnrnph1-211 & $-10,61,576,893$ & ENSMUST00000155282.8 & Myo5a-214 & $9,842,528,635$ \\
\hline ENSMUST00000107857.10 & Ap2a1-202 & $-10,25,950,022$ & ENSMUST00000097864.8 & Pum1-203 & $9,697,390,548$ \\
\hline ENSMUST00000194801.5 & Rbm5-224 & $-10,17,739,189$ & ENSMUST00000212451.1 & Mau2-206 & $9,650,005,064$ \\
\hline ENSMUST00000132947.1 & Pds5b-204 & $-9,920,365,984$ & ENSMUST00000217647.1 & Scaper-205 & $9,631,678,893$ \\
\hline ENSMUST00000154403.7 & Polg-214 & $-9,869,641,974$ & ENSMUST00000212100.1 & lqsec1-210 & $9,270,515,836$ \\
\hline ENSMUST00000170647.1 & Tnpo3-209 & $-9,791,146,902$ & ENSMUST00000039892.8 & Tbc1d25-201 & $9,251,526,431$ \\
\hline ENSMUST00000231973.1 & D16Ertd472e-205 & $-9,770,163,529$ & ENSMUST00000183148.7 & Ank3-239 & $9,209,545,896$ \\
\hline ENSMUST00000095037.1 & Whrn-204 & $-9,756,960,752$ & ENSMUST00000163483.1 & Rab1a-206 & $9,145,085,159$ \\
\hline ENSMUST00000208730.1 & Picalm-212 & $-9,589,780,073$ & ENSMUST00000230614.1 & Acap2-203 & $9,047,789,318$ \\
\hline ENSMUST00000066986.12 & Zfp142-202 & $-9,460,284,761$ & ENSMUST00000171937.1 & Arhgap35-202 & $6,619,772,687$ \\
\hline ENSMUST00000222395.1 & Atg2b-205 & $-9,411,773,318$ & ENSMUST00000205765.1 & Crebbp-205 & $6,563,438,613$ \\
\hline ENSMUST00000150905.1 & Htra1-204 & $-9,363,035,965$ & ENSMUST00000224799.1 & Spire1-207 & $5,741,134,073$ \\
\hline ENSMUST00000092614.8 & Pcgf1-201 & $-9,362,877,176$ & ENSMUST00000098816.9 & Slc7a2-202 & $4,478,565,603$ \\
\hline ENSMUST00000216284.1 & Cep164-207 & $-4,150,389,783$ & ENSMUST00000194877.5 & Ints7-206 & $4,424,334,603$ \\
\hline
\end{tabular}

The twenty most highly downregulated (Log2(Fold Change) $<0$ ) and twenty most highly upregulated (Log2(Fold Change) $>0$ ) Differentially Expression Transcripts (DET-with a false discovery rate $(\mathrm{FDR})<0,05)$ modulated in $\mathrm{C} 2 \mathrm{C} 12$ cells treated with RGMa during differentiation. Access number and transcript name identified in the Ensembl database; log2(FD) $<0$ corresponds to the fold change of the downregulation and log2(FD) $>0$, of the upregulation of each transcript after RGMa treatment

\section{Types of DE RNA}

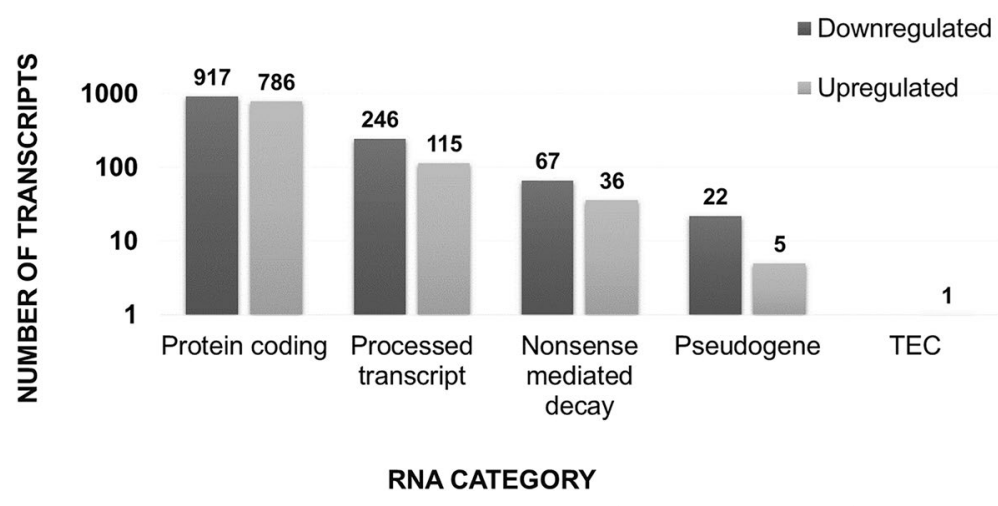

Fig. 2 RNA biotypes modulated by RGMa treatment. RGMa could modulate the differential expression of 13 RNA biotypes, classified in six RNA categories according to Ensembl (https://m.ensembl.org/info/genome/genebuild/biotypes.html): (1) protein coding, (2) processed transcripts (IncRNA: antisense, bidirection-promoter-IncRNA, lincRNA, retained intron and ncRNA: snRNA and Mt-rRNA), (3) nonsense mediated decay, (4) pseudogenes (processed-pseudogenes, transcribed-processed-pseudogene, and unprocessed-pseudogene), and (5) Tec (to be experimentally confirmed)

to 'metabolism' and 'tissue survival' were the most downregulated after RGMa treatment. 'Purine nucleoside triphosphate metabolic process', 'peptide biosynthetic process,' 'translation,' and 'positive regulation of apoptotic signaling pathway' were the most enriched terms of biological processes (Fig. 5A). Cellular components were mostly associated with 'mitochondria protein complex,' 'actin cytoskeleton,' 'cytosolic ribosome', 


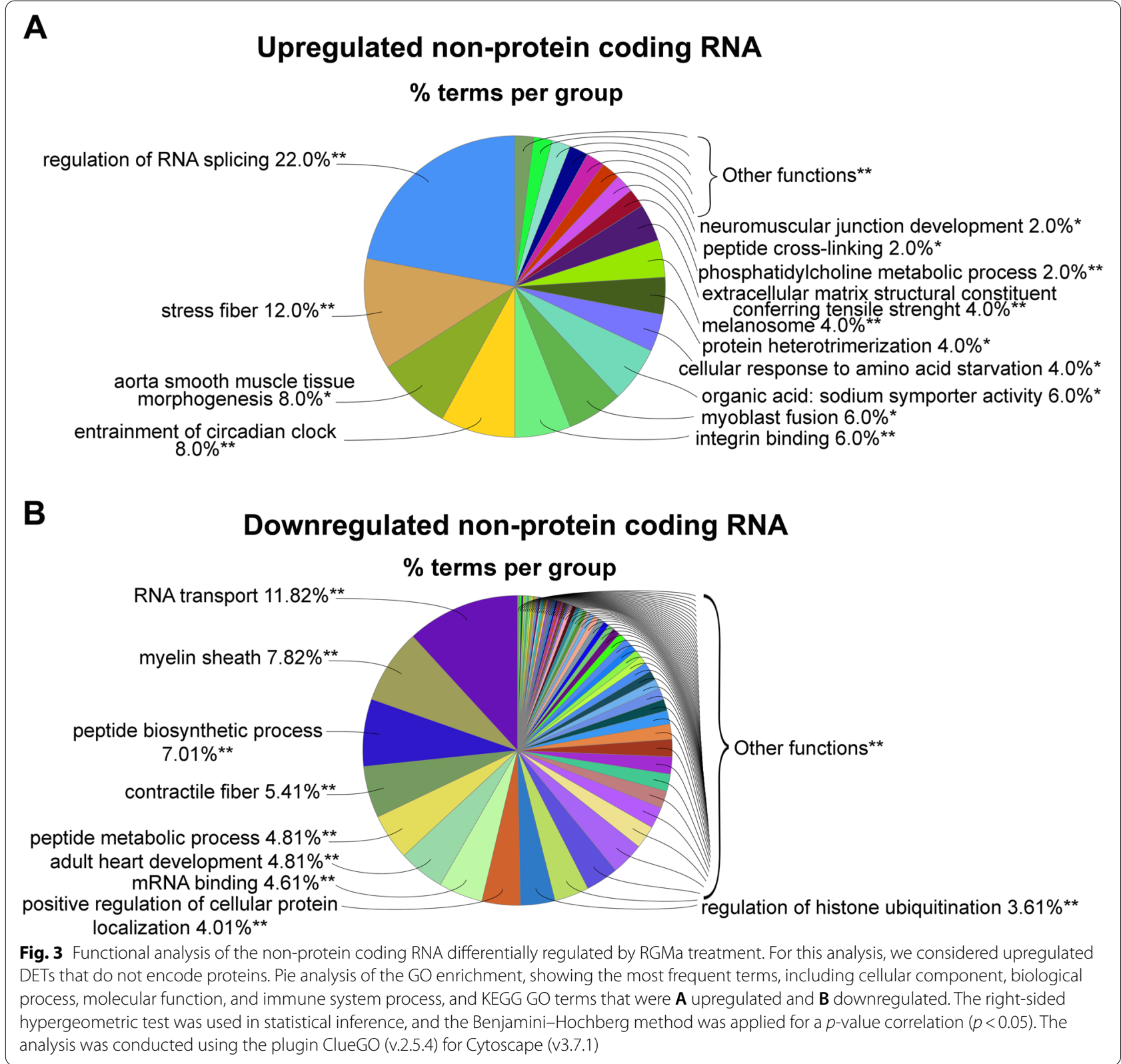

'proteasome complex,' and 'vesicle coat' (Fig. 5B), while those found to be associated with molecular function were grouped in 'activity of nucleoside-triphosphatase,' 'ATPase', and 'positive regulation of catalysis' categories (Fig. 5C).

\section{Cell adhesion and hypertrophy-associated terms}

We selected GO terms associated with "cell adhesion" and with "skeletal muscle structure" and "hypertrophy", from both up and downregulated DETs, for a network analysis (Fig. 6).

\section{(See figure on next page.)}

Fig. 4 Functional analysis of the protein coding RNA upregulated by RGMa. For this analysis, we considered the DETs that encode proteins that were found to be upregulated (FC > 1) by the treatment with RGMa, compared to the control. A-C Pie chart analysis of the three $\mathrm{GO}$ categories used to classify the upregulated protein coding transcripts. The right-sided hypergeometric test was used in statistical inference, and the BenjaminiHochberg method was applied for a $p$-value correlation $(p<0.0001)$. The analysis was conducted using the plugin ClueGO (v.2.5.4) for Cytoscape (v3.7.1) 
A

\section{Biological Process}

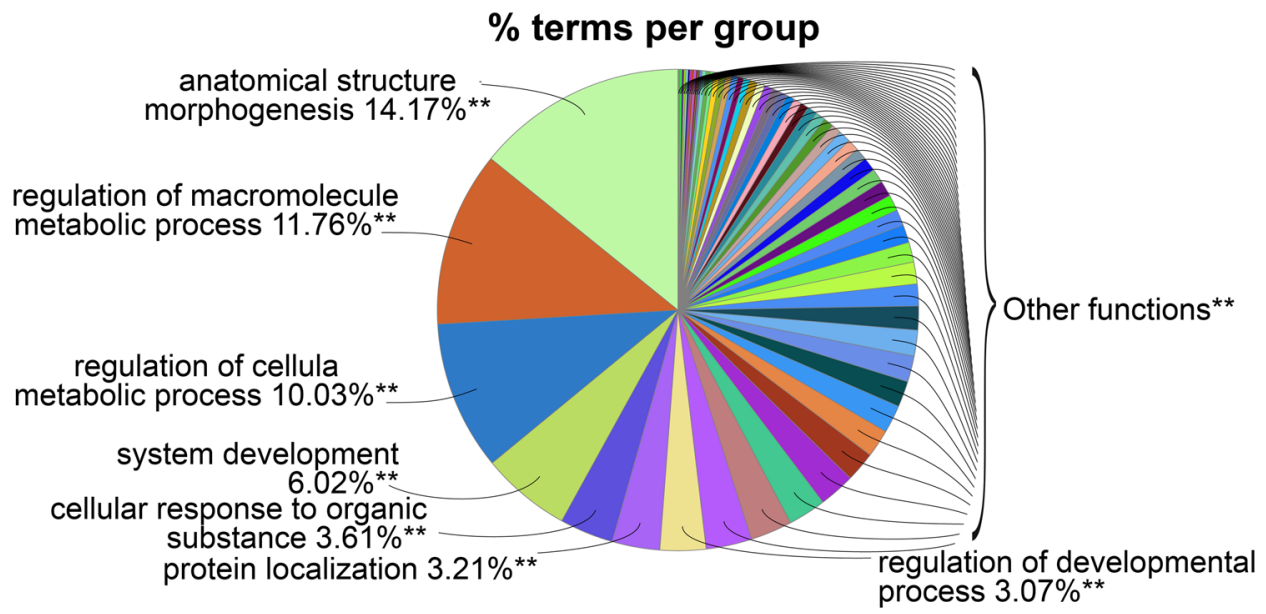

B

\section{Cellular Component}

$\%$ terms per group

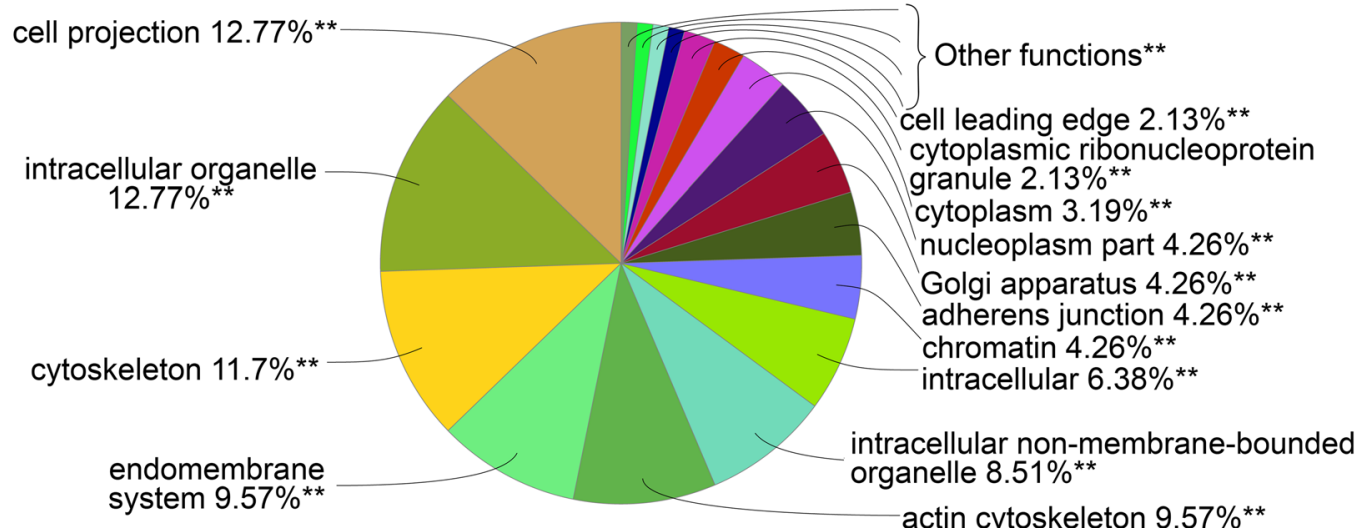

C

\section{Molecular Function \\ $\%$ terms per group}

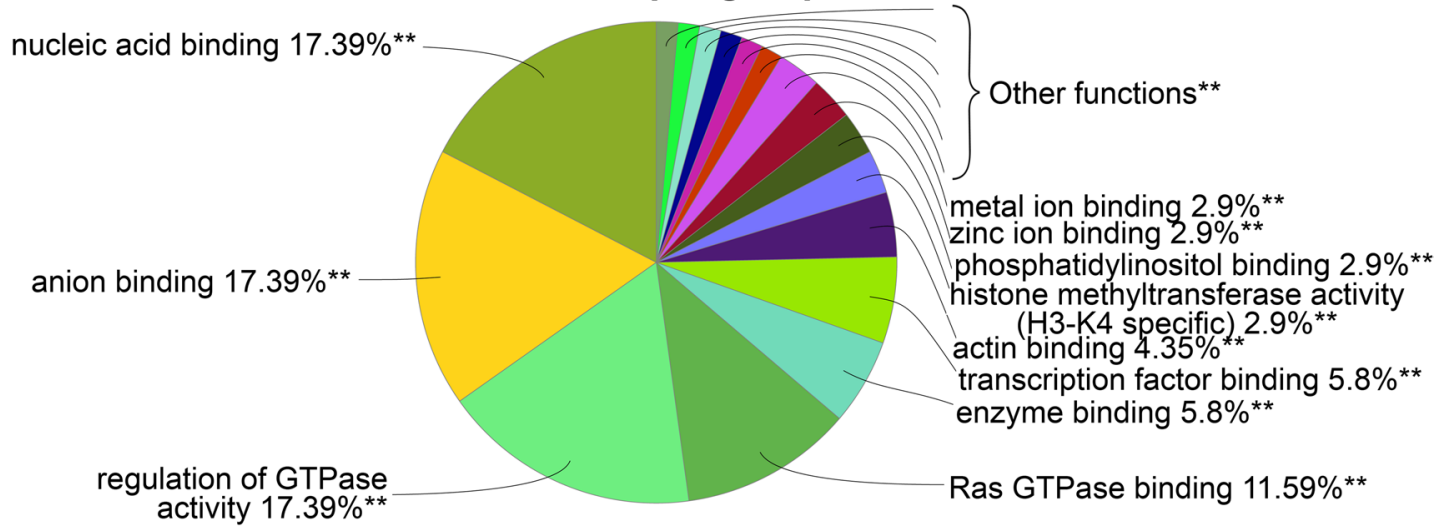

Fig. 4 (See legend on previous page.) 
Our analysis revealed a number of upregulated transcripts were associated with GO terms including 'adherens junctions', 'cell-substrate adherens junction', 'adherens junction assembly' and 'adherens junction organization', 'cell-substrate junction assembly', 'regulation of cell adhesion', 'extracellular matrix organization', 'collagen-containing extracellular matrix'; while downregulated transcripts were mainly associated with 'Focal adhesion,' 'regulation of cell-substrate adhesion', 'cell-substrate adhesion', 'cellmatrix adhesion' and 'tight junction' (Fig. 6A).

Related to muscle term, we could find upregulated transcripts associated with GO terms including 'contractile fiber part', 'muscle cell differentiation' and 'striated muscle cell differentiation'; while the transcripts found as downregulated were mostly associated with the following GO terms: 'sarcomere', 'Z disk', 'I band,' 'myofibril', 'contractile fiber,' 'myofilament', 'striated muscle thin filament', 'troponin complex,' 'actin cytoskeleton', 'contractile actin filament bundle,' 'stress fiber', among others (Fig. 6B).

\section{RNA-seq validation}

We chose 12 DET isoforms to validate our RNA-seq data and analysis by qPCR. Arhgap35-202 and 201, Hipk2206, Mef2d-202, 204 and 203, mTOR-202, Myh9-201, Myo5a-214, Nfat5-206, 208 and 214, Parva-203, and Pou2f1-208 were selected from the upregulated DETs isoforms, and Cep164-170, Kifc3-202 and Pcgf1-201 isoforms were chosen from the downregulated ones. The qPCR results showed a total concordance with the RNAseq analysis (Fig. 7).

\section{Discussion}

Although originally identified as a guidance clue for axonal growth, RGMa has been identified as playing roles in a number of different biological processes, including during myogenesis. $R G M a$ transcripts could be found in chicken somites at the origin site of the muscle and satellite cell precursors [24]. In adult muscle, RGMa was found in regions of the sarcolemma and sarcoplasm, with an expression pattern similar to sarcomeric proteins [25]. Initial functional studies revealed that RGMa can induce myonuclear accretion and hypertrophy of myotubes, suggesting that this axon guidance molecule might be involved with the mechanisms that modulate skeletal muscle cell size [25].
However, the molecular mechanisms induced by RGMa during these important muscle phenotypes have not been clarified thus far. RGMa exerts its canonical effects through the type-I transmembrane neogenin receptor [6, 7, 9, 37], but it can also work as a bone morphogenetic protein (BMP) co-receptor, as it shares the same binding site in BMP-R1A with BMP ligands [15]. Notably, both signaling pathways seem to be active in skeletal muscle cells, inducing similar phenotypes in controlling the cell size, but these effects were never investigated in the context of having RGMa as a possible ligand. Using RGMa recombinant proteins in $\mathrm{C} 2 \mathrm{C} 12$ cells, we could not clearly elucidate if RGMa effects were induced via neogenin and/or BMP signaling pathways, possibly because these receptors do not have RGMa as an exclusive ligand [38]. For this reason, in this work, the transcriptome of $\mathrm{C} 2 \mathrm{C} 12$ cells was sequenced after being treated with RGMa recombinant protein during the late differentiation stage to detect the transcripts that had their expression modulated by this axon guidance molecule during the differentiation of skeletal muscle cells.

A database composed of 23,856 transcripts expressed during $\mathrm{C} 2 \mathrm{C} 12$ differentiation was generated. Sequenced biological triplicates from treated and control groups were found to be homogeneous, conferring internal consistency and reproducibility of replicate samples. Three technical replicates are considered a sufficient for a reliable quantitative inferential analysis [39]. From these expressed transcripts, 2,195 were modulated by RGMa treatment, with 943 upregulated and 1,252 downregulated.

From this database, it was noted that RGMa was able to modulate the expression of five RNA biotypes. The most frequent RNA biotype modulated by RGMa was 'protein coding', which included ORF containing transcripts. However, a significant portion of DETs were included in categories involved with the regulation of gene expression, including in the 'nonsense mediated decay' (composed of transcripts with a premature stop codon) and 'processed transcript' (composed of 'retained intron RNA', 'antisense' and 'ncRNA') biotypes. According to Wong et al. (2013), a number of transcripts must be destroyed to permit developmental transitions during differentiation [40]. Therefore, this data suggests that RGMa treatment induced

(See figure on next page.)

Fig. 5 Functional analysis of the protein coding RNA downregulated by RGMa. For this analysis, we considered the DETs that encode proteins and were found to be downregulated ( $F C<1$ ) in the RGMa treated group, compared to the control one. A-C Pie chart analysis of the three GO categories for downregulated DETs. D Functionally grouped network of enriched categories for expressed transcripts, annotated for 'biological process,"cellular component,' and 'molecular function' GO terms. The right-sided hypergeometric test was used in statistical inference, and the Benjamini-Hochberg method was applied for a $p$-value correlation $(p<0.001)$. The analysis was conducted using the plugin ClueGO (v.2.5.4) for Cytoscape (v3.7.1) 


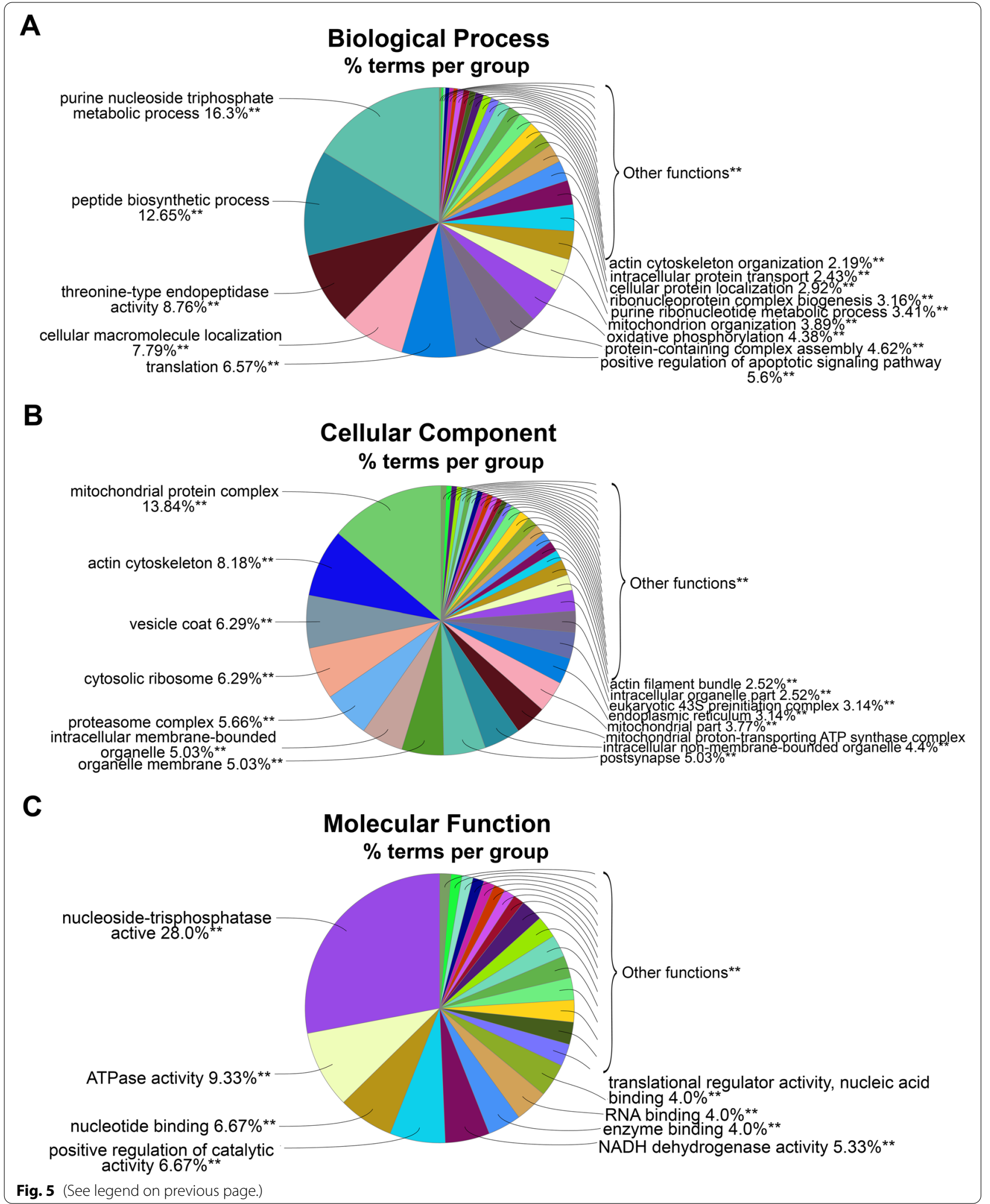




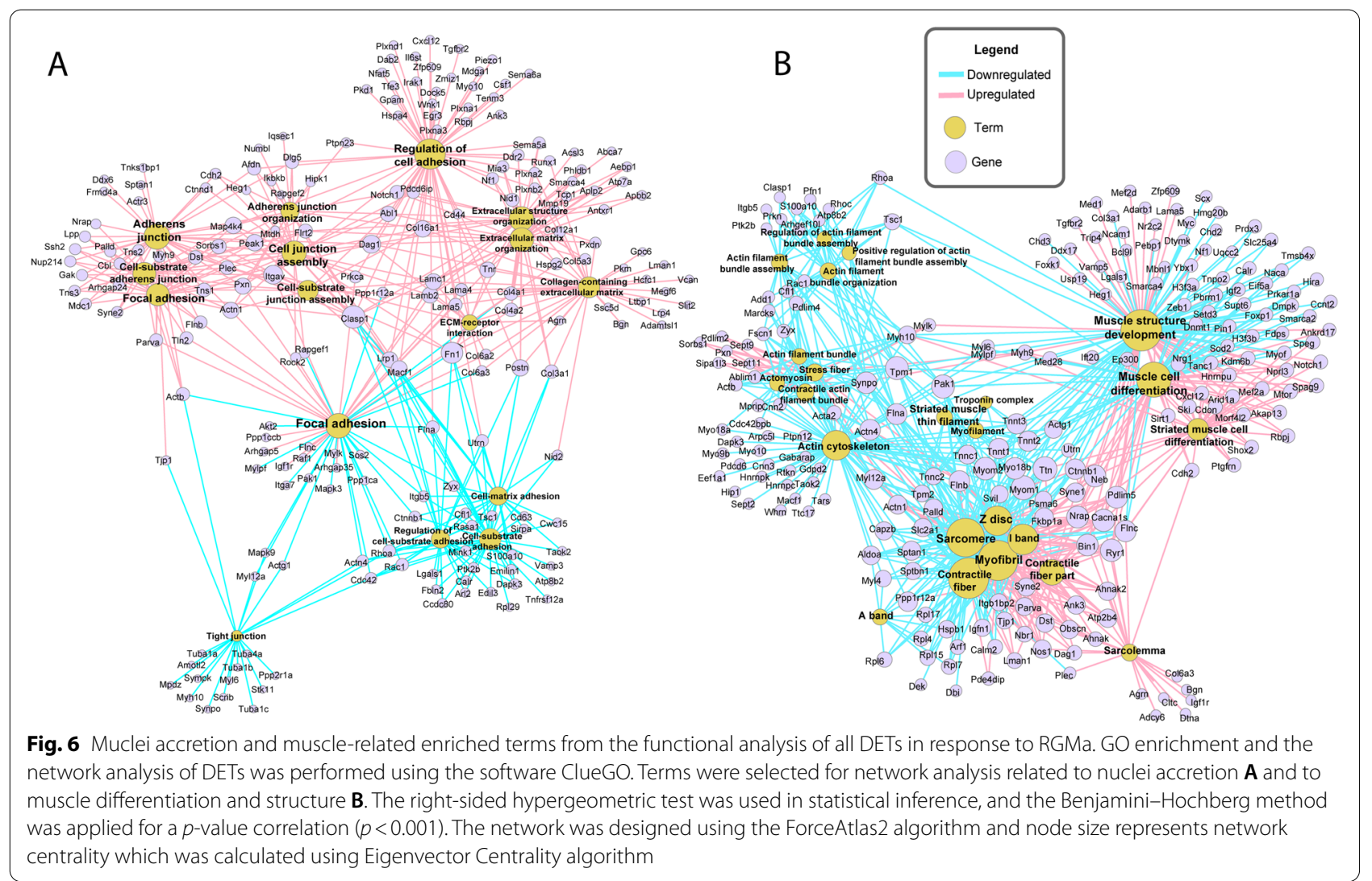

\section{RT_qPCR of RNAseq expression profile}

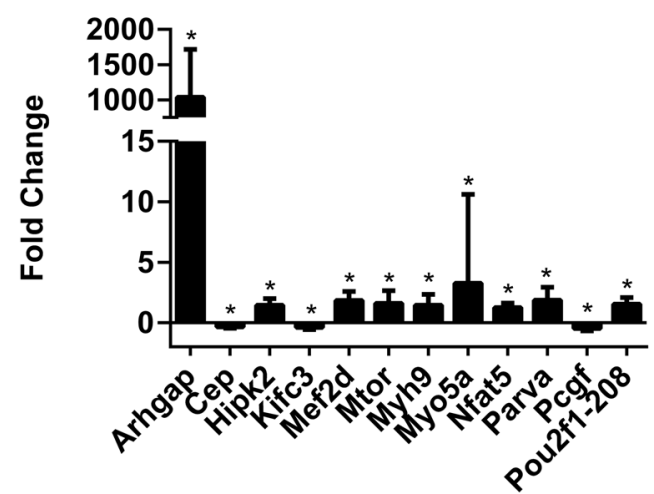

Fig. 7 Validation of the RNA-seq expression profiles by $\mathrm{QPCR}$. A subset of twelve DETs that were upregulated and downregulated by RGMa treatment during muscle differentiation were used to validate the obtained RNA-seq expression data. Transcripts were selected by their expression and their known association with muscle hyperplasic or hypertrophic phenotypes. Expression patterns indicate agreement between the two methods and ${ }^{*}$, significance of $p$-adj $<0.05$ the regulation of the genes that were being expressed during the differentiation stages using these particular molecular mechanisms, allowing the adaptation of these cells to reach terminal differentiation.

Additionally, our analysis also revealed that RGMa could differentially induce the expression of alternatively spliced transcripts. Pou $2 F 1$ (Pou Class 2 Homeobox 1, also known as Oct-1) isoforms were found to be the most upregulated DET (Pou2f1-208, ENSMUST00000160260.9), as well as the most downregulated DET (Pou2f1-205, ENSMUST00000111427.9) by RGMa treatment in skeletal muscle cells. Although the specific functions of each of these isoforms have not been described thus far, it is known that Pou2F1 is an ubiquitously expressed member of the Pou transcription factor family and is associated with a plethora of processes, including the activation of some snRNA, histone $\mathrm{H} 2 \mathrm{~B}$, immunoglobulins, and other housekeeping genes [41], the regulation of the circadian clock [42], and glycolytic metabolism [43]. In skeletal muscle cells, this transcription factor was associated with the activation of pro-inflammatory immune response in patients with myalgia [44] and with MyHC IIB expression, when associated with MEF2 and the serum response factor (SRF) [45-47]. Pou2F1 was also identified on a slow 
skeletal muscle troponin I promoter in Gaoyou duck skeletal muscle [48]. In addition to Pou 2F1, multiple isoforms for myoferlin (Myof), myosin heavy chain 10 (Myh10), myosin IXB (Myo9b), titin (Ttn), tensin 2 (Tns2), supervillain (Svil), and chromodomain helicase DNA binding protein 2 (Chd2) were also found as modulated by the RGMa treatment in $\mathrm{C} 2 \mathrm{C} 12$ cells; these genes are of wide importance for development, differentiation, and maintenance of skeletal muscle cells.

\section{RGMa treatment modulated the expression of muscle hypertrophic markers}

The protein coding DETs were analyzed to determine how RGMa induces hypertrophic and nuclear accretion effects on skeletal muscle cells.

Our transcriptome database showed that RGMa induced the expression of the mammalian target of rapamycin $(m T O R)$ transcript, which is a common factor from different pathways that culminate with skeletal muscle hypertrophy [27, 49-53]. RGMa could specifically induce $m T O R$ transcript isoform 202 (ENSMUST00000103221.10), suggesting a new mechanism for this isoform in these cells. The effect of RGMa on mTOR expression was also confirmed by qPCR. mTOR exerts its effects as part of two complexes, termed mTORC1 and mTORC2. Increased mTORC1 activity can positively regulate muscle protein synthesis via S6K1 and also inhibit its negative regulation when working via $4 \mathrm{EBP} 1[27,54]$. TSC1, in a complex with TSC2, is responsible for the negative regulation of $\mathrm{mTORC} 1$ signaling, inhibiting the nutrientmediated or growth factor-stimulated phosphorylation of S6K1 and 4EBP1 [53]. Furthermore, TSC1-204 (ENSMUST00000113870.3) was also highly downregulated by RGMa treatment in skeletal muscle cells. The inhibition of TSC1/2 protein synthesis resulted in rapid activation of mTORC1 signaling independent of Akt $[53,55]$. The hypertrophic effects observed by RGMa treatment could then be a result of the inhibition of the TSC1 transcript and of the induction of $m T O R$ expression, which are both crucial for muscle growth. Additionally, although the TSC1/2 complex is not physically associated with mTORC1, it is required for mTORC2 activation and consequently, for Akt phosphorylation, in a manner that is independent of its GTPase-activating protein activity toward Rheb [56]. Thus, the inhibition of TSC1 by RGMa suggests that RGMa simultaneously works to prevent mTORC2 activation. The fact that TSC1 inhibition contributes to mTORC1 activation independently of Akt, as well as to mTORC2 inhibition, resulting in the loss of Akt stimulation [55], might explain why Akt was not induced by RGMa in skeletal muscle cells. Our results suggest that mTOR upregulation in response to RGMa is independent of Akt phosphorylation.
Other factors associated with the mTORC pathway were also dysregulated by the RGMa treatment and could contribute to including this axon guidance molecule in an alternative muscle hypertrophic pathway. For example, RGMa could induce the expression of the phospholipase D1 (Pld1-202, ENSMUST00000120834.8) transcript, which was found to be an activator of mTORC1 $[50,57]$.

RGMa could also induce the upregulation of members of the Myocyte Enhancer Factor 2 (Mef2) family, specifically Mef2a-204 (ENSMUSG00000030557.17) and Mef2d-204 (ENSMUSG00000001419.17) isoforms. Mef2 transcription factors activate many muscle-specific growth factor-induced genes and regulate muscle cell differentiation and muscle embryonic development [58-60]. Mef2 can also act as a nodal point for remodeling programs in metabolic gene expression, fiber-type switching, and skeletal muscle regeneration [58, 59, 61]. Mef $2 a$ upregulation can also contribute to terminal differentiation and myoblast fusion, which is also consistent with the present GO term analysis and with the RGMa muscle phenotype [25, 38]. Mef2a, Mef2c, and Mef2d deleted in combination in satellite cells abolished skeletal muscle regeneration after cardiotoxin injury [59].

Our RNA-seq database suggested other hypertrophic mechanisms that could be regulated by RGMa treatment, including the upregulation of Sirtuin 1 (Sirt1), which is known to regulate protein degradation via FoxO inhibition [62]; the upregulation of Nos1, which interacts with Sirt1 [63]; or the downregulation of genes that promote muscle protein degradation, such as the activating transcription factor 4 (Atf4) $[64,65]$.

\section{RGMa treatment also modulated the expression of genes associated with nuclei accretion}

We have also searched for genes associated with myonuclear accretion that were modulated by RGMa treatment in $\mathrm{C} 2 \mathrm{C} 12$ cells. Among these, cadherin2 (Cdh2, ENSMUST00000025166.13), integrin alpha-V (Itgav, ENSMUST00000141725.2), neural cell adhesion molecule (NCAM, ENSMUST00000166811.8), calcium voltage-gated channel subunit alpha1S (Cacna1s, ENSMUST00000112068.9), actinin alpha 1 (Actn1, ENSMUST00000167327.1), disabled homolog 2 (Dab2, ENSMUST00000080880.11), myoferlin (Myof, ENSMUST00000224560.1, ENSMUST00000041475.15, ENSMUST00000224518.1), the myosins Myo5a (ENSMUST00000155282.8, ENSMUST00000123128.7), Myo10 (ENSMUST00000022882.11, ENSMUST00000110457.7, ENSMUST00000125667.2), Myh9 (ENSMUST00000016771.12), Myh10 (ENSMUST00000102611.9) phosphatase, and actin regulator 4 (Phactr4, ENSMUST00000136711.1) were upregulated by RGMa treatment. 
Myof, for example, is a member of the Ferlin protein family, highly expressed in myoblasts during the prefusion phase of differentiation and in myofibers, especially during regeneration after injury [66-68]. It is associated with fusion events and intracellular trafficking in muscle, including myoblast fusion, vesicle traffic, membrane repair, and endocytic recycling [69].

$M y h 9$ and Myh10 are equally fundamental for the positive regulation of cell-cell adhesion and myoblast fusion [70]. Myh9 is known as non-muscle myosin heavy chain IIa (NMMHC-IIA), while Myh10 is the non-muscle myosin heavy chain IIb [71]. These myosins are expressed in most cell types, working as motor proteins in a variety of processes requiring contractile force, such as cytokinesis, cell migration, polarisation and adhesion, maintenance of cell shape, and signal transduction [72-74]. In skeletal muscle cells, non-muscle myosins drive myoblasts to align and fuse to form multinucleated myotubes [70, 75]. The knockdown of these myosins inhibit the change of the myoblast shape, interfering with cell-cell adhesion and fusion [70].

Dab2 plays an important role as a modulator of cellcell interactions, as it is a clathrin adaptor and can mediate integrin signaling [76]. In the musculature, Dab2 was detected during early myogenic differentiation [77, 78]. Shang et al. (2020) showed that Dab2 expression is upregulated in $\mathrm{C} 2 \mathrm{C} 12$ myoblast during the differentiation in myotubes, and its knockdown resulted in reduced myoblast fusion and fewer myotubes. Besides, Dab2 overexpression could enhance the myotube formation and also restore the myotube differentiation capacity of its knockdown [79].

The calcium voltage-gated channel subunit alpha1 S (Cacna1s-202, ENSMUST00000112068.10) encodes one of the five subunits of the L-type voltage-dependent calcium channel in skeletal muscle cells. In the musculature, calcium is generally related to muscle contraction and muscle relaxation [80-82]. However, the regulation of calcium influx into muscle cells plays a critical role in muscle differentiation [82, 83]. Intracellular calcium is able to regulate transcription factors necessary for myotube fusion [83, 84], while its reduction inhibits myoblast differentiation [85]. The upregulation of Cacna1s in response to RGMa treatment suggests an association with the regulation of intracellular calcium, which is important for the myoblast fusion process and myotube contraction.

\section{Conclusion}

The current work allowed us to unravel some molecular mechanisms that were altered in skeletal muscle cells after treatment with RGMa, especially those associated with muscle nuclei accretion and hypertrophy.
Our analysis suggested that RGMa induced cell hypertrophy via (i) upregulation of hypertrophic markers, (ii) downregulation of inhibitors of hypertrophic pathways, (iii) downregulation of transcripts related to the positive regulation of muscle atrophy, and (iv) upregulation of transcripts that negatively regulate atrophy. At the same time, transcripts associated with known myoblast fusion pathways were also found to be modulated by RGMa, mainly those related to cell-cell adhesion pathways.

Our results provide comprehensive knowledge of skeletal muscle transcriptional changes and pathways in response to RGMa treatment.

\section{Material and methods}

Cell culture and differentiation

The lineage of immortalized mouse myoblasts $\mathrm{C} 2 \mathrm{C} 12$ (ATCC $^{\circledR} \mathrm{CRL} 17722^{\mathrm{TM}}$ ) was cultured at $37{ }^{\circ} \mathrm{C}$ and $5 \% \mathrm{CO}_{2}$ in growth medium (GM), composed of DMEM (Dulbecco's Modified Eagle's Medium) with high glucose and L-glutamine (Gibco), supplemented with $10 \%$ fetal bovine serum (FBS, Gibco) and 1\% penicillin, streptomycin, and amphotericin B solution (Gibco). Myogenic differentiation was induced in differentiation medium (DM), composed of DMEM, supplemented with $2 \%$ horse serum (Gibco) and 1\% penicillin, streptomycin, and amphotericin B. For growth or differentiation conditions, the medium was replaced every 2 days.

\section{RGMa recombinant protein treatment}

C2C12 cells were seeded at $2 \times 10^{4}$ cells per well in 24-well plates and cultivated in $\mathrm{GM}$ at $37^{\circ} \mathrm{C}$ and $5 \% \mathrm{CO}_{2}$. After reaching $90-100 \%$ confluency, cells were induced to differentiate in DM for $72 \mathrm{~h}$ (Fig. 1A). DM was then replaced with fasting medium (FM), composed of DMEM supplemented with $0.2 \% \mathrm{FBS}$, and cells were incubated at the same conditions for $3 \mathrm{~h}$. Subsequently, C2C12 were treated with $50 \mathrm{ng} / \mathrm{ml}$ mouse RGMa recombinant protein (R\&D Systems) in FM and incubated for an additional $48 \mathrm{~h}$, as previously described [38]. The recombinant protein was omitted in the control samples.

\section{Total RNA isolation and CDNA synthesis}

Cells were harvested in TriReagent (Sigma Aldrich) as pools of three wells in triplicate. Total RNA isolation was performed according to the manufacturer's instructions. Sample integrity, purity, and concentration were evaluated by electrophoresis in $1 \%$ agarose gel and in NanoDrop ${ }^{\circledR}$ ND-1000 UV/Vis Spectrophotometer, respectively.

The quality of the total RNA was also evaluated in a Bioanalyzer (Agilent) before being submitted to sequencing. Values for RNA integrity number (RIN) ranging from 8 to 10 were considered suitable for RNA-seq. 


\section{RNA-seq library preparation and next-generation sequencing (NGS)}

For cDNA library construction, $2 \mu \mathrm{g}$ of total RNA were treated with $1 \mathrm{U}$ of DNaseI amplification grade (Invitrogen) and purified according to the TruSeq Stranded mRNA Sample Prep LS Protocol of Illumina (http://grcf.jhmi.edu/hts/ protocols/mRNA-Seq_SamplePrep_1004898_D.pdf), using magnetic microspheres for messenger RNA separation. The purified mRNA was fragmented in Illumina buffer. Superscript III (Invitrogen) and oligo(dT) were used for reverse transcription of the first cDNA strand. The second strand was synthesized using the enzymes RNase $\mathrm{H}$ and DNA Polymerase I (Illumina). Molecule ends were treated with T4 DNA Polymerase and Klenow DNA Polymerase (Illumina), making them blunt. The 3' end of the synthetized cDNA was phosphorylated with T4 PNK (Illumina) and adenylated with Klenow exo (Illumina). Adaptors were bound to cDNA ends, and the samples were purified and selected by size of $200 \mathrm{bp} \pm 25 \mathrm{bp}$ after fractioning in agarose gel electrophoresis (QIAquick Gel Extraction Kit, QIAGEN). Purified cDNA was quantified by RT-qPCR using adaptor-specific oligonucleotides (Illumina).

Sequencing was performed using HiScanSQ (Illumina),according to the manufacturer's recommendations, and using the paired-end reads protocol. Each sample was sequenced until it reached around 34 million reads/library.

\section{Mapping RNA-seq data}

Transcript quantification analysis was performed based on Salmon (version 0.13.1), an open-source and freely-licensed software (available at https://github.com/COMBINE-lab/ Salmon [86]). Raw reads were used as an input to quantify transcripts in mapping-based mode. The current version of the mouse transcriptome (available at https://www.genco degenes.org/mouse/release_M20.html) was used as a reference, which includes all RNA categories used to classify the transcripts obtained in this work.

\section{Statistical RNA-seq}

Statistical analysis was performed using the DESeq2 package of $\mathrm{R}$ Bioconductor [87]. An adjusted $p$-value with a false discovery rate (FDR) correction (Benjamini and Hochberg, 1995) of 5\% was calculated and used to control false-positive significance in transcript expression variation. $\log 2$ (fold change) $>0$ and $\log 2$ (fold change) $<0$ were selected as the threshold to show an increase or decrease in transcript expression of treated groups relative to the control group.

Transcript expression pattern and RNA-seq quality analysis Transcript-specific normalisation was performed to remove disparities in the base means correlations and to eliminate the noise of transcripts with low expression.
Normalised transcripts were plotted in MA form using the DESeq2 package to generate a scatter plot of $\log 2$ fold changes $<0$ and $>0$ versus the mean of normalised counts of transcripts, considering DE those with FDR $<0.05$. The correlation of each sample and the clustering of the treated and control groups was performed by calculating the PCC of normalised read-counts.

\section{Functional RNA-seq analysis}

The GO enrichment and the network analysis of DETs was performed using the software ClueGO v.2.5.4 [88] and Gephi v 0.9.2 (https://ojs.aaai.org/index.php/ ICWSM/article/view/13937). The right-sided hypergeometric test was used to identify overrepresented GO terms and the BenjaminiHochberg method was used for the correction of the $p$-values $(p<0.001)$. The Ensembl Transcript ID of the DETs was used as input for ClueGO analysis. Terms were selected for network analysis by related to nuclei accretion (Fig. 6A) and related to muscle differentiation and structure (Fig. 6B). The network was designed using the ForceAtlas 2 algorithm and node size represents network centrality which was calculated using Eigenvector Centrality algorithm.

The heatmap graph was obtained using the D3Heatmap package (https://www.rdocumentation.org/packa ges/d3heatmap/versions/0.6.1.2), using ID ensemble transcripts as an input (of clue go output for muscle associated terms) and the correlated base mean expression.

\section{Primer design and qPCR}

qPCR was performed for the specific upregulated isoforms of Arhgap35-202 and 201, Hipk2-206, Mef2d-202, 204 and 203, mTOR-202, Myh9-201, Myo5a-214, Nfat5206, 208 and 214, Parva-203, Pou2f1-208 and for the downregulated isoforms of Cep164-170, Kifc3-202 and Pcgf1-201, that were selected due to their importance for muscle phenotypes, as well as by their relevance between the more enriched terms.

The multiline interface (http://multalin.toulouse.inra. $\mathrm{fr} /$ multalin/) was used for the alignment of genes with some specific isoforms up and others downregulated by RGMa. The non-consensus sequences among them were selected to avoid undesired isoforms and the consensus ones were used to obtain an amplicon of up to $250 \mathrm{bp}$ for the chosen isoforms. Primer 3.0 software was used for primer design. Manual primers were designed for small specific strings.

cDNA was synthesized using $1 \mu$ g of total RNA following the recommendations of the RevertAid ${ }^{\mathrm{TM}} \mathrm{H}$ Minus First Strand cDNA Synthesis kit (Fermentas). 
qPCR was performed in the Rotor-Gene RT-qPCR system (Qiagen), using the iTaq Universal Sybr Green Supermix (Bio Rad) and 0.4-0.8 $\mu \mathrm{M}$ of each primer for a final volume of $10 \mu \mathrm{l}$. GAPDH was used as a housekeeping gene. The analysis of differential gene expression was performed using REST 2009 (Relative Expression Software Tool, V.2.0.13) software via randomisation tests (Pair Wise Fixed Reallocation Randomisation Test) [89] with $95 \%$ significance.

\section{Abbreviations}

4EBP1: Ukaryotic Translation Initiation Factor 4E Binding Protein 1; Acap: ArfGAP With Coiled-Coil, Ankyrin Repeat And PH Domains 1; Actn1: Actinin alpha 1; Akt: Serine/Threonine Kinase 1; ALK5: Activin A Receptor Type II-Like Protein Kinase Of 53kD, also Known as Transforming Growth Factor Beta Receptor 1; Ank3: Ankyrin 3; Ap2a1: Adaptor Related Protein Complex 2 Subunit Alpha 1; Arhgap-35: Rho GTPase Activating Protein 35; Atf4: Activating transcription factor 4; BMP: Bone Morphogenetic protein; BMP-R1A: BMP type I receptor A; C2C12: Immortalized Mouse Myoblast cell line; Cacna1s: Calcium voltagegated channel subunit alpha1S; Cep164: Centrosomal Protein 164; Cdh2: Cadherin2; cDNA: Complementary DNA; Chd2: Chromodomain helicase DNA binding protein 2; C-RGMa: RGMa C-terminal domain; Dab2: Disabled homolog 2; DE: Differentially expressed; DET: Differentially expressed transcripts; DM: Differentiation medium; DMEM: Dulbecco's Modified Eagle's Medium; FBS: Fetal bovine serum; FDR: False Discovery Rate; FM: Fasting medium; FoxO: Forkhead Box Protein O; GDPH: Glyceraldehyde 3-phosphate dehydrogenase; GM: Growth medium; GO: Gene Ontology; GPI-anchor: Glycosylphosphatidylinositol-anchor; GTP: Nucleotide guanosine triphosphate; Hipk2: Homeodomain Interacting Protein Kinase 2; Iqsec1: IQ Motif And Sec7 Domain ArfGEF 1; Itgav: Integrin alpha-V; KEGG: Kyoto Encyclopedia of Genes and Genomes; Kifc3: Kinesin Family Member C3; IncRNA: Long non coding RNA; Mau2: MAU2 Sister Chromatid Cohesion Factor; Mef2: Myocyte Enhancer Factor 2; mRNA: Messenger RNA; mTOR: Mechanistic Target Of Rapamycin Kinase; mTORC1/2: Mechanistic target of rapamycin complex 1/2; Myh9: Myosin heavy chain 9; Myh 10: Myosin heavy chain 10; Myof: Myoferlin; Myhc IIB: Myosin heavy chain Ilb; Myo5a: Myosin VA; NCAM: Neural cell adhesion molecule; NCBI: National Center for Biotechnology Information; ncRNA: Non coding RNA NGS: Next Generation Sequencing; NMD: Nonsense Mediated Decay; Nos1: Nitric Oxide Synthase 1; Nrap: Nebulin Related Anchoring Protein; N-RGMa: RGMa N-terminal domain; Oct-1: Octamer-Binding Transcription Factor 1 Oligo(dT): Oligonucleotide (deoxythymine); ORF: Open reading frame; Parva: Parvin Alpha; PCC: Pearson correlation coefficient; Pcgf1: Polycomb Group Ring Finger 1; Phactr4: Phosphatase and actin regulator 4; Picalm: Phosphatidylinositol Binding Clathrin Assembly Protein; Pld1: Phospholipase D1; Pou2F1: Octamer-Binding Transcription Factor-1, also known as Oct1; Rab1a: RAB1A, Member RAS Oncogene Family; Rac: Family Small GTPase 1; RGD: Tripeptide Arg-Gly-Asp; RGMa: Repulsive Guidance Molecule a; Rho: Rhodopsin; RIN: RNA integrity number; RNA: Ribonucleic acid; RNA-seq: RNA sequencing; RT-qPCR: Quantitative reverse transcription polymerase chain reaction; S6k1: Ribosomal Protein S6 Kinase B1; Scaper: S-Phase Cyclin A Associated Protein In The ER, also known as Zinc Finger Protein 291; Sirt1: Sirtuin; Smad2/3: Mothers Against Decapentaplegic Homolog 2/3; snRNA: Small nuclear RNA; SRF: Serum response factor; Svil: Supervillain; T4 PNK: T4 Polynucleotide Kinase; Tns2:Tensin2; Ttn:Titin; Tbc1d25: TBC1 Domain Family Member 25; TEC: To be Experimentally Confirmed; TGF $\beta 1$ : Transforming Growth Factor $\beta 1$; Tnpo3: Transportin 3; Tsc1: Tuberous Sclerosis 1 Protein; Tsc2: Tuberin.

\section{Supplementary Information}

The online version contains supplementary material available at https://doi. org/10.1186/s12864-022-08396-w.

Additional file1: Supplementary file 1. Differentially expressed transcripts modulated by RGMa treatment during the differentiation of $\mathrm{C} 2 \mathrm{C} 12$ cells.

Additional file 2: Supplementary file 2. Differentially expressed genes modulated by RGMa treatment during the differentiation of $\mathrm{C} 2 \mathrm{C} 12$ cell.

\section{Acknowledgements}

We wish to thank Professor Antonio Figueira from Centro de Energia Nuclear na Agricultura (CENA, Universidade de São Paulo, Piracicaba, Brazil) for supporting our laboratory.

\section{Authors' contributions}

For this research, AGLC and ECJ designed the study. AGLC, JMN, and ACC performed the in vitro and qPCR experiments. LLC generated the RNA-seq data, and LEDB, PHAC and IMCAC performed the bioinformatic analysis. AGLC and IGDS extracted the biological information of the expression data. AGLC and ECJ wrote the manuscript. ECJ supervised and administrated the study. GABS provided part of the funding acquisition and supervised experimental work. All authors have read and agreed to the published version of the manuscript.

\section{Funding}

This research was supported by the Coordenação de Aperfeiçoamento de Pessoal de Nível Superior (CAPES), the Conselho Nacional de Desenvolvimento Científico e Tecnológico (CNPq) and the Fundação de Amparo à Pesquisa do Estado de Minas Gerais (FAPEMIG). AGLC, ACC and JMN were financed by the Coordenação de Aperfeiçoamento de Pessoal de Nível Superior (CAPES), Finance Code 001. ECJ and LLC received a scholarship from CNPq.

\section{Availability of data and materials}

The raw transcriptome sequencing data (RNA-seq) from the technical replicates of C2C12 myoblast treated with RGMa are available under the NCBI-BioProject submission code PRJNA730936. The datasets supporting the conclusions of this article are included within the article and its additional files.

\section{Declarations}

Ethics approval and consent to participate

Not applicable.

\section{Consent for publication}

Not applicable.

\section{Competing interests}

The authors declare that they have no competing interests.

\section{Author details}

'Departamento de Morfologia, Instituto de Ciências Biológicas, Universidade Federal de Minas Gerais, Av Antonio Carlos, 6627, Pampulha, Belo Horizonte, Minas Gerais 31.270-901, Brasil. ${ }^{2}$ Departamento de Zootecnia, Escola Superior de Agricultura Luiz de Queiroz, Universidade de São Paulo, Piracicaba, Brasil. ${ }^{3}$ Departamento de Botânica, Instituto de Ciências Biológicas, Universidade Federal de Minas Gerais, Belo Horizonte, Brasil. ${ }^{4}$ Departamento de Ciências Naturais, Universidade Federal de São João del Rei, São João del Rei, Brasil.

${ }^{5}$ Departamento de Bioquímica E Imunologia, Instituto de Ciências Biológicas, Universidade Federal de Minas Gerais, Belo Horizonte, Brasil.

Received: 22 June 2021 Accepted: 15 February 2022

Published online: 07 March 2022

\section{References}

1. Monnier PP, Sierra A, Macchi P, Deitinghoff L, Andersen JS, Mann M, Flad M, Hornberger MR, Stahl B, Bonhoeffer F, et al. RGM is a repulsive guidance molecule for retinal axons. Nature. 2002;419(6905):392-5.

2. Stahl B, Müller B, von Boxberg Y, Cox EC, Bonhoeffer F. Biochemical characterization of a putative axonal guidance molecule of the chick visual system. Neuron. 1990;5(5):735-43.

3. Müller B, Jay D, Bonhoeffer F. Chromophore-assisted laser inactivation of a repulsive axonal guidance molecule. Curr Biol. 1996;6(11):1497-502.

4. Siebold C, Yamashita T, Monnier PP, Mueller BK, Pasterkamp RJ. RGMs: structural insights, molecular regulation, and downstream signaling. Trends Cell Biol. 2017;27(5):365-78.

5. Zhou Z, Xie J, Lee D, Liu Y, Jung J, Zhou L, Xiong S, Mei L, Xiong WC. Neogenin regulation of BMP-induced canonical Smad signaling and endochondral bone formation. Dev Cell. 2010;19(1):90-102. 
6. Harada K, Fujita Y, Yamashita T. Repulsive guidance molecule A suppresses angiogenesis. Biochem Biophys Res Commun. 2016;469(4):993-9.

7. Fujita Y, Yamashita T. The roles of RGMa-neogenin signaling in inflammation and angiogenesis. Inflamm Regen. 2017:37:6.

8. Nohra R, Beyeen AD, Guo JP, Khademi M, Sundqvist E, Hedreul MT, Sellebjerg F, Smestad C, Oturai AB, Harbo HF, et al. RGMA and IL21R show association with experimental inflammation and multiple sclerosis. Genes Immun. 2010;1 1(4):279-93.

9. Rajagopalan S, Deitinghoff L, Davis D, Conrad S, Skutella T, Chedotal A, Mueller BK, Strittmatter SM. Neogenin mediates the action of repulsive guidance molecule. Nat Cell Biol. 2004;6(8):756-62.

10. Itokazu T, Fujita Y, Takahashi R, Yamashita T. Identification of the neogenin-binding site on the repulsive guidance molecule A. PLoS One. 2012;7(3):e32791.

11. Fitzgerald DP, Seaman C, Cooper HM. Localization of Neogenin protein during morphogenesis in the mouse embryo. Dev Dyn. 2006;235(6):1720-5.

12. Fitzgerald DP, Cole SJ, Hammond A, Seaman C, Cooper HM. Characterization of neogenin-expressing neural progenitor populations and migrating neuroblasts in the embryonic mouse forebrain. Neuroscience. 2006;142(3):703-16

13. Cole SJ, Bradford D, Cooper HM. Neogenin: A multi-functional receptor regulating diverse developmental processes. Int J Biochem Cell Biol. 2007;39(9):1569-75.

14. Bell CH, Healey E, van Erp S, Bishop B, Tang C, Gilbert RJC, Aricescu AR, Pasterkamp RJ, Siebold C. Structure of the repulsive guidance molecule (RGM)-neogenin signaling hub. Science. 2013;341(6141):77-80.

15. Healey EG, Bishop B, Elegheert J, Bell CH, Padilla-Parra S, Siebold C. Repulsive guidance molecule is a structural bridge between neogenin and bone morphogenetic protein. Nat Struct Mol Biol. 2015;22(6):458-65.

16. Babitt JL, Zhang Y, Samad TA, Xia Y, Tang J, Campagna JA, Schneyer AL, Woolf CJ, Lin HY. Repulsive guidance molecule (RGMa), a DRAGON homologue, is a bone morphogenetic protein co-receptor. J Biol Chem. 2005;280(33):29820-7.

17. Samad TA, Rebbapragada A, Bell E, Zhang Y, Sidis Y, Jeong SJ, Campagna JA, Perusini S, Fabrizio DA, Schneyer AL, et al. DRAGON, a bone morphogenetic protein co-receptor. J Biol Chem. 2005;280(14):14122-9.

18. Babitt JL, Huang FW, Wrighting DM, Xia Y, Sidis Y, Samad TA, Campagna JA, Chung RT, Schneyer AL, Woolf CJ, et al. Bone morphogenetic protein signaling by hemojuvelin regulates hepcidin expression. Nat Genet. 2006;38(5):531-9.

19. Corradini E, Babitt JL, Lin HY. The RGM/DRAGON family of BMP coreceptors. Cytokine Growth Factor Rev. 2009:20(5-6):389-98.

20. Zhang AS, West AP Jr, Wyman AE, Bjorkman PJ, Enns CA. Interaction of hemojuvelin with neogenin results in iron accumulation in human embryonic kidney 293 cells. J Biol Chem. 2005;280(40):33885-94.

21. Zhang AS, Yang F, Wang J, Tsukamoto H, Enns CA. Hemojuvelin-neogenin interaction is required for bone morphogenic protein-4-induced hepcidin expression. J Biol Chem. 2009;284(34):22580-9.

22. Tian C, Liu J. Repulsive guidance molecules (RGMs) and neogenin in bone morphogenetic protein (BMP) signaling. Mol Reprod Dev. 2013;80(9):700-17.

23. Zhang R, Wu Y, Xie F, Zhong Y, Wang Y, Xu M, Feng J, Charish J, Monnier PP, Qin X. RGMa mediates reactive astrogliosis and glial scar formation through TGFbeta1/Smad2/3 signaling after stroke. Cell Death Differ. 2018:25(8):1503-16.

24. Jorge EC, Ahmed MU, Bothe I, Coutinho LL, Dietrich S. RGMa and RGMb expression pattern during chicken development suggest unexpected roles for these repulsive guidance molecules in notochord formation, somitogenesis, and myogenesis. Dev Dyn. 2012;241(12):1886-900.

25. Martins AF, Xavier Neto J, Azambuja A, Sereno ML, Fiqueira A, Campos-Junior PH, Rosario MF, Toledo CB, Silva GA, Kitten GT, et al. Repulsive Guidance Molecules a, b and c Are Skeletal Muscle Proteins, and Repulsive Guidance Molecule a Promotes Cellular Hypertrophy and Is Necessary for Myotube Fusion. Cells Tissues Organs. 2015;200(5):326-38.

26. Sartorelli V, Fulco M. Molecular and cellular determinants of skeletal muscle atrophy and hypertrophy. Sci STKE. 2004;2004(244):re11.

27. Bonaldo P, Sandri M. Cellular and molecular mechanisms of muscle atrophy. Dis Model Mech. 2013;6(1):25-39.
28. Sartori R, Schirwis E, Blaauw B, Bortolanza S, Zhao J, Enzo E, Stantzou A, Mouisel E, Toniolo L, Ferry A, et al. BMP signaling controls muscle mass. Nat Genet. 2013;45(11):1309-18.

29. Otto A, Patel K. Signaling and the control of skeletal muscle size. Exp Cell Res. 2010;316(18):3059-66.

30. Lyons GE, Moore R, Yahara O, Buckingham ME, Walsh FS. Expression of NCAM isoforms during skeletal myogenesis in the mouse embryo. Dev Dyn. 1992;194(2):94-104.

31. Nishi M, Yasue A, Nishimatu S, Nohno T, Yamaoka T, Itakura M, Moriyama K, Ohuchi H, Noji S. A missense mutant myostatin causes hyperplasia without hypertrophy in the mouse muscle. Biochem Biophys Res Commun. 2002;293(1):247-51.

32. Krauss RS, Cole F, Gaio U, Takaesu G, Zhang W, Kang JS. Close encounters: regulation of vertebrate skeletal myogenesis by cell-cell contact. J Cell Sci. 2005;118(Pt 11):2355-62.

33. Krauss RS. Regulation of promyogenic signal transduction by cell-cell contact and adhesion. Exp Cell Res. 2010;316(18):3042-9.

34. Krauss RS, Joseph GA, Goel AJ. Keep your friends close: cell-cell contact and skeletal myogenesis. Cold Spring Harb Perspect Biol. 2017:9(2):a029298.

35. McClure MJ, Ramey AN, Rashid M, Boyan BD, Schwartz Z. Integrin-alpha7 signaling regulates connexin $43, \mathrm{M}$-cadherin, and myoblast fusion. Am J Physiol Cell Physiol. 2019;316(6):C876-87.

36. Bastos UMC, de Andrade Rosa I, Teixeira JD, Goncalves G, Costa ML, Quintas LEM, Mermelstein C. Isoproterenol induces an increase in muscle fiber size by the proliferation of Pax7-positive cells and in a mTOR-independent mechanism. Cell Biol Int. 2019;43(12):1425-34.

37. De Vries M, Cooper HM. Emerging roles for neogenin and its ligands in CNS development. J Neurochem. 2008:106(4):1483-92.

38. do Carmo Costa A, Copola AGL, Carvalho ESC, Nogueira JM, Silva GAB, Jorge EC. RGMa can induce skeletal muscle cell hyperplasia via association with neogenin signaling pathway. In Vitro Cell Dev Biol Anim. 2021:57(4):415-27.

39. Conesa A, Madrigal P, Tarazona S, Gomez-Cabrero D, Cervera A, McPherson A, Szczesniak MW, Gaffney DJ, Elo LL, Zhang X, et al. A survey of best practices for RNA-seq data analysis. Genome Biol. 2016;17:13.

40. Wong JJ, Ritchie W, Ebner OA, Selbach M, Wong JW, Huang Y, Gao D, Pinello N, Gonzalez M, Baidya K, et al. Orchestrated intron retention regulates normal granulocyte differentiation. Cell. 2013;154(3):583-95.

41. Segil N, Roberts SB, Heintz N. Mitotic phosphorylation of the Oct-1 homeodomain and regulation of Oct-1 DNA binding activity. Science. 1991;254(5039):1814-6.

42. Bozek K, Relogio A, Kielbasa SM, Heine M, Dame C, Kramer A, Herzel H. Regulation of clock-controlled genes in mammals. PLoS One. 2009;4(3):e4882.

43. Stepchenko AG, Lyanova BM, Krylova ID, Ilyin YV, Georgieva SG, Pankratova EV. Differentiation of monocytic cells is accompanied by a change in the expression of the set of Oct-1 isoforms. Dokl Biochem Biophys. 2018:483(1):306-8.

44. Elam MB, Majumdar G, Mozhui K, Gerling IC, Vera SR, Fish-Trotter H, Williams RW, Childress RD, Raghow R. Patients experiencing statin-induced myalgia exhibit a unique program of skeletal muscle gene expression following statin re-challenge. PLoS One. 2017;12(8):e0181308.

45. Lakich MM, Diagana TT, North DL, Whalen RG. MEF-2 and Oct-1 bind to two homologous promoter sequence elements and participate in the expression of a skeletal muscle-specific gene. J Biol Chem. 1998;273(24):15217-26.

46. Bhavsar PK, Dellow KA, Yacoub MH, Brand NJ, Barton PJ. Identification of cis-acting DNA elements required for expression of the human cardiac troponin I gene promoter. J Mol Cell Cardiol. 2000;32(1):95-108.

47. Allen DL, Weber JN, Sycuro LK, Leinwand LA. Myocyte enhancer factor-2 and serum response factor binding elements regulate fast Myosin heavy chain transcription in vivo. J Biol Chem. 2005;280(17):17126-34.

48. Ji GG, Shu JT, Zhang M, Ju XJ, Shan YJ, Liu YF, Tu YJ. Transcriptional regulatory region and DNA methylation analysis of TNNI1 gene promoters in Gaoyou duck skeletal muscle (Anas platyrhynchos domestica). Br Poult Sci. 2019;60(3):202-8.

49. Bodine SC, Stitt TN, Gonzalez M, Kline WO, Stover GL, Bauerlein R, Zlotchenko E, Scrimgeour A, Lawrence JC, Glass DJ, et al. Akt/mTOR pathway is a crucial regulator of skeletal muscle hypertrophy and can prevent muscle atrophy in vivo. Nat Cell Biol. 2001;3(11):1014-9. 
50. Hong-Brown $L Q$, Brown $C R$, Navaratnarajah $M$, Lang $C H$. Activation of AMPKTTSC2/PLD by alcohol regulates MTORC1 and mTORC2 assembly in C2C12 myocytes. Alcohol Clin Exp Res. 2013;37(11):1849-61.

51. Schiaffino S, Dyar KA, Ciciliot S, Blaauw B, Sandri M. Mechanisms regulating skeletal muscle growth and atrophy. FEBS J. 2013;280(17):4294-314.

52. Sartori R, Sandri M. BMPs and the muscle-bone connection. Bone. 2015;80:37-42

53. Zhan J, Chitta RK, Harwood FC, Grosveld GC. Phosphorylation of TSC2 by PKC-delta reveals a novel signaling pathway that couples protein synthesis to mTORC1 activity. Mol Cell Biochem. 2019;456(1-2):123-34.

54. Laplante M, Sabatini DM. mTOR signaling in growth control and disease. Cell. 2012;149(2):274-93.

55. Zhang $\mathrm{H}$, Cicchetti G, Onda H, Koon HB, Asrican K, Bajraszewski N, Vazquez F, Carpenter CL, Kwiatkowski DJ. Loss of Tsc1/Tsc2 activates mTOR and disrupts PI3K-Akt signaling through downregulation of PDGFR. J Clin Invest. 2003;112(8):1223-33.

56. Huang J, Dibble CC, Matsuzaki M, Manning BD. The TSC1-TSC2 complex is required for proper activation of mTOR complex 2. Mol Cell Biol. 2008;28(12):4104-15.

57. Xu L, Salloum D, Medlin PS, Saqcena M, Yellen P, Perrella B, Foster DA. Phospholipase $D$ mediates nutrient input to mammalian target of rapamycin complex 1 (mTORC1). J Biol Chem. 2011;286(29):25477-86.

58. Potthoff MJ, Olson EN. MEF2: a central regulator of diverse developmental programs. Development. 2007;134(23):4131-40.

59. Liu N, Nelson BR, Bezprozvannaya S, Shelton JM, Richardson JA, BasselDuby R, Olson EN. Requirement of MEF2A, C, and D for skeletal muscle regeneration. Proc Natl Acad Sci U S A. 2014;111(11):4109-14.

60. Schiaffino S, Dyar KA, Calabria E. Skeletal muscle mass is controlled by the MRF4-MEF2 axis. Curr Opin Clin Nutr Metab Care. 2018;21(3):164-7.

61. Yuan $H$, Niu Y, Liu X, Fu L. Exercise increases the binding of MEF2A to the $\mathrm{Cpt} 1 \mathrm{~b}$ promoter in mouse skeletal muscle. Acta Physiol (Oxf). 2014;212(4):283-92.

62. Lee D, Goldberg AL. SIRT1 protein, by blocking the activities of transcription factors FoxO1 and FoxO3, inhibits muscle atrophy and promotes muscle growth. J Biol Chem. 2013;288(42):30515-26.

63. Koltai E, Bori Z, Chabert C, Dubouchaud H, Naito H, Machida S, Davies KJ, Murlasits Z, Fry AC, Boldogh I, et al. SIRT1 may play a crucial role in overload-induced hypertrophy of skeletal muscle. J Physiol. 2017;595(11):3361-76.

64. Ebert SM, Monteys AM, Fox DK, Bongers KS, Shields BE, Malmberg SE, Davidson BL, Suneja M, Adams CM. The transcription factor ATF4 promotes skeletal myofiber atrophy during fasting. Mol Endocrinol. 2010;24(4):790-9.

65. Ebert SM, Dyle MC, Bullard SA, Dierdorff JM, Murry DJ, Fox DK, Bongers KS, Lira VA, Meyerholz DK, Talley JJ, et al. Identification and Small Molecule Inhibition of an Activating Transcription Factor 4 (ATF4)-dependent Pathway to Age-related Skeletal Muscle Weakness and Atrophy. J Biol Chem. 2015;290(42):25497-511.

66. Davis DB, Delmonte AJ, Ly CT, MCNally EM. Myoferlin, a candidate gene and potential modifier of muscular dystrophy. Hum Mol Genet. 2000;9(2):217-26.

67. Doherty KR, Cave A, Davis DB, Delmonte AJ, Posey A, Earley JU, Hadhazy $M$, McNally EM. Normal myoblast fusion requires myoferlin. Development. 2005;132(24):5565-75.

68. Demonbreun AR, Lapidos KA, Heretis K, Levin S, Dale R, Pytel P, Svensson EC, MCNally EM. Myoferlin regulation by NFAT in muscle injury, regeneration and repair. J Cell Sci. 2010;123(Pt 14):2413-22.

69. Posey AD Jr, Demonbreun A, McNally EM. Ferlin proteins in myoblast fusion and muscle growth. Curr Top Dev Biol. 2011;96:203-30.

70. Swailes NT, Colegrave M, Knight PJ, Peckham M. Non-muscle myosins 2A and $2 \mathrm{~B}$ drive changes in cell morphology that occur as myoblasts align and fuse. J Cell Sci. 2006;119(Pt 17):3561-70.

71. Simons M, Wang M, McBride OW, Kawamoto S, Yamakawa K, Gdula D, Adelstein RS, Weir L. Human nonmuscle myosin heavy chains are encoded by two genes located on different chromosomes. Circ Res. 1991;69(2):530-9.

72. Foth BJ, Goedecke MC, Soldati D. New insights into myosin evolution and classification. Proc Natl Acad Sci U S A. 2006;103(10):3681-6.

73. Even-Ram S, Yamada KM. Of mice and men: Relevance of cellular and molecular characterizations of myosin IIA to MYH9-related human disease. Cell Adh Migr. 2007;1(3):152-5.
74. Sebe-Pedros A, Grau-Bove X, Richards TA, Ruiz-Trillo I. Evolution and classification of myosins, a paneukaryotic whole-genome approach. Genome Biol Evol. 2014;6(2):290-305.

75. Dey SK, Saha S, Das P, Das MR, Jana SS. Regulation of nonmuscle myosin II during 3-methylcholanthrene induced dedifferentiation of $\mathrm{C2} 212$ myotubes. Exp Cell Res. 2014;326(1):68-77.

76. Margadant C, Monsuur HN, Norman JC, Sonnenberg A. Mechanisms of integrin activation and trafficking. Curr Opin Cell Biol. 2011;23(5):607-14.

77. Tomczak KK, Marinescu VD, Ramoni MF, Sanoudou D, Montanaro F, Han M, Kunkel LM, Kohane IS, Beggs AH. Expression profiling and identification of novel genes involved in myogenic differentiation. FASEB J. 2004;18(2):403-5.

78. Lee EJ, Malik A, Pokharel S, Ahmad S, Mir BA, Cho KH, Kim J, Kong JC, Lee DM, Chung KY, et al. Identification of genes differentially expressed in myogenin knock-down bovine muscle satellite cells during differentiation through RNA sequencing analysis. PLoS One. 2014;9(3):e92447.

79. Shang N, Lee JTY, Huang T, Wang C, Lee TL, Mok SC, Zhao H, Chan WY. Disabled-2: a positive regulator of the early differentiation of myoblasts. Cell Tissue Res. 2020;381(3):493-508.

80. Bannister RA. Bridging the myoplasmic gap: recent developments in skeletal muscle excitation-contraction coupling. J Muscle Res Cell Motil. 2007:28(4-5):275-83.

81. Nakada T, Kashihara T, Komatsu M, Kojima K, Takeshita T, Yamada M. Physical interaction of junctophilin and the CaV1.1 C terminus is crucial for skeletal muscle contraction. Proc Natl Acad Sci U S A. 2018;115(17):4507-12.

82. Park JW, Lee JH, Kim SW, Han JS, Kang KS, Kim SJ, ParkTS. Muscle differentiation induced up-regulation of calcium-related gene expression in quail myoblasts. Asian-Australas J Anim Sci. 2018;31(9):1507-15.

83. Tajhya RB, Hu X, Tanner MR, Huq R, Kongchan N, Neilson JR, Rodney GG, Horrigan FT, Timchenko LT, Beeton C. Functional KCa1.1 channels are crucial for regulating the proliferation, migration and differentiation of human primary skeletal myoblasts. Cell Death Dis. 2016;7(10):e2426.

84. Liu JH, Konig S, Michel M, Arnaudeau S, Fischer-Lougheed J, Bader CR, Bernheim L. Acceleration of human myoblast fusion by depolarization: graded Ca2+ signals involved. Development. 2003;130(15):3437-46.

85. Porter GA Jr, Makuck RF, Rivkees SA. Reduction in intracellular calcium levels inhibits myoblast differentiation. J Biol Chem. 2002;277(32):28942-7.

86. Patro R, Duggal G, Love MI, Irizarry RA, Kingsford C. Salmon provides fast and bias-aware quantification of transcript expression. Nat Methods. 2017;14(4):417-9

87. Love MI, Huber W, Anders S. Moderated estimation of fold change and dispersion for RNA-seq data with DESeq2. Genome Biol. 2014;15(12):550.

88. Bindea G, Mlecnik B, Hackl H, Charoentong P, Tosolini M, Kirilovsky A, Fridman WH, Pages F, Trajanoski Z, Galon J. ClueGO: a Cytoscape plug-in to decipher functionally grouped gene ontology and pathway annotation networks. Bioinformatics. 2009;25(8):1091-3.

89. Pfaffl MW, Horgan GW, Dempfle L. Relative expression software tool (REST) for group-wise comparison and statistical analysis of relative expression results in real-time PCR. Nucleic Acids Res. 2002;30(9):e36.

\section{Publisher's Note}

Springer Nature remains neutral with regard to jurisdictional claims in published maps and institutional affiliations. 